\title{
Lipid Accumulation Product is Related to Metabolic Syndrome in Women with Polycystic Ovary Syndrome
}

Authors

Affiliation

\author{
S. Xiang, F. Hua, L. Chen, Y. Tang, X. Jiang, Z. Liu
}

Department of Endocrinology. the Third Affiliated Hospital of Suzhou University, Changzhou, Jiangsu, China
Key words

polycystic ovary syndrome

- metabolic syndrome

- lipid accumulation product

received 19.07.2012

first decision 19.07.2012

accepted $\quad 21.12 .2012$

Bibliography

DOI http://dx.doi.org/

10.1055/s-0032-1333261

Exp Clin Endocrinol Diabetes

2013; 121: 115-118

(c) J. A. Barth Verlag in

Georg Thieme Verlag KG

Stuttgart · New York

ISSN 0947-7349

\section{Correspondence}

\section{F. Hua}

Department of Endocrinology The Third Affiliated Hospital of Suzhou University

Changzhou 213003

Jiangsu

China

Tel.: + 86/519/6887 1183

Fax: + 86/517/8662 1235

czhuafei@vip.sina.com

\begin{abstract}
$\boldsymbol{\nabla}$

Purpose: Metabolic disturbances are common features of polycystic ovary syndrome (PCOS), which possibly enhance the risk of diabetes and cardiovascular disease. Lipid accumulation product (LAP) is an emerging cardiovascular risk factor. The aim of this study was to explore the ability of LAP to identify metabolic syndrome (MS) in PCOS women.

Methods: In a cross-sectional study, anthropometric, biochemical and clinical parameters were measured in 105 PCOS women. Receiver operating characteristic (ROC) analysis was used to find
\end{abstract}

\section{Introduction}

\section{$\nabla$}

Polycystic ovary syndrome (PCOS) is one of the most common female endocrine disorders with a prevalence of $5-10 \%$ in women of reproductive age [1]. It is characterized by hyperandrogenemia, chronic anovulation, and polycystic ovary morphology [2,3].The etiology of PCOS is still not very clear, however, previous studies have shown that insulin resistance (IR) and central obesity are key pathological factors of PCOS [4,5]. PCOS is not only the reproductive endocrine disease, but also metabolic disorder. Patients with PCOS are often accompanied by metabolic abnormalities, such as dyslipidemia, obesity, and glucose intolerance, which are also components of the metabolic syndrome (MS) [6].

MS is a cluster of metabolic disorders associated with increased risk of cardiovascular disease (CVD), and central or abdominal obesity is considered a fundamental pathology for the MS. Many of the anthropometric and metabolic abnormalities of PCOS overlap with components of MS, and PCOS patients are at increased risk of having MS [7]. Adults with MS are at a greater risk of developing CVD [8]. PCOS women with MS may be at even greater risk for CVD because they out the cut-off points of LAP to predict MS. MS was categorized according to International Diabetes Federation (IDF) criteria.

Results: The prevalence of MS was $43.8 \%$ in this study. PCOS women with MS had significantly higher LAP levels compared to those without MS. LAP was highly correlated with components of MS. ROC analysis showed that LAP was a significant discriminator for MS in PCOS women, and the optimal cutoff point of LAP to predict MS was 54.2 (93.3\% sensitivity, $96.7 \%$ specificity).

Conclusions: LAP seems to be associated with MS and has a strong and reliable diagnostic accuracy for MS in PCOS women. are exposed to risk factors at a younger age [9]. Early and accurate identification of high-risk individuals for MS could be important to predict and prevent CVD and type 2 diabetes. There is, therefore, an urgent need to develop a simple, accurate and economic predictor for MS in PCOS. The lipid accumulation product (LAP), which was first described by Kahn [10], is based on a combination of waist circumference (WC) and serum triglycerides (TG) and serves as a simple index for lipid over accumulation in adults. High levels of LAP are associated with CVD, impaired glucose tolerance (IGT) and type 2 diabetes [11,12]. In healthy population, LAP is a powerful marker of MS $[13,14]$, however, to the best of our knowledge, there is currently limited research on the relationship between LAP and MS in women with PCOS. Thus, we aim to investigate the ability of LAP to identify MS in PCOS patients.

\section{Materials and Methods $\nabla$} Patients

From February 2009 to October 2011, 105 patients diagnosed with PCOS at the Department of Endocrinology of the Third Affiliated Hospital 
Table 1 Clinical characteristics of PCOS women with or without MS.

\begin{tabular}{|c|c|c|c|}
\hline Variables & $\begin{array}{l}\text { Without MS } \\
(n=60)\end{array}$ & $\begin{array}{l}\text { With MS } \\
(n=45)\end{array}$ & $P$-values \\
\hline age (years) & $24.6 \pm 4.6$ & $25.2 \pm 5.1$ & 0.478 \\
\hline BMI $\left(\mathrm{Kg} / \mathrm{m}^{2}\right)$ & $22.7 \pm 1.7$ & $27.2 \pm 2.2$ & $<0.001$ \\
\hline waist $(\mathrm{cm})$ & $71.2 \pm 4.3$ & $85.2 \pm 3.6$ & $<0.001$ \\
\hline $\begin{array}{l}\text { systolic blood pressure } \\
(\mathrm{mmHg})\end{array}$ & $118 \pm 8$ & $131 \pm 9$ & $<0.001$ \\
\hline $\begin{array}{l}\text { diastolic blood pressure } \\
(\mathrm{mmHg})\end{array}$ & $72 \pm 5$ & $81 \pm 9$ & $<0.001$ \\
\hline fasting glucose (mmol/L) & $5.2 \pm 0.4$ & $5.7 \pm 0.5$ & $<0.001$ \\
\hline $\begin{array}{l}\text { postload glucose } \\
(\mathrm{mmol} / \mathrm{L})\end{array}$ & $7.4 \pm 1.1$ & $8.7 \pm 1.5$ & $<0.001$ \\
\hline fasting insulin (mIU/L) & $18.5 \pm 4.1$ & $25.5 \pm 4.9$ & $<0.001$ \\
\hline HOMA-IR & $4.35 \pm 1.27$ & $6.62 \pm 1.64$ & $<0.001$ \\
\hline triglycerides (mmol/L) & $2.02 \pm 0.65$ & $3.48 \pm 0.86$ & $<0.001$ \\
\hline $\begin{array}{l}\text { total cholesterol } \\
(\mathrm{mmol} / \mathrm{L})\end{array}$ & $4.88 \pm 0.33$ & $5.18 \pm 0.39$ & $<0.05$ \\
\hline LDL cholesterol (mmol/L) & $2.44 \pm 0.27$ & $2.56 \pm 0.31$ & 0.065 \\
\hline HDL cholesterol (mmol/L) & $1.19 \pm 0.12$ & $0.94 \pm 0.07$ & $<0.001$ \\
\hline $\begin{array}{l}\text { luteinizing hormone } \\
(\mathrm{mlU} / \mathrm{ml})\end{array}$ & $15.22 \pm 2.27$ & $16.32 \pm 2.36$ & $<0.05$ \\
\hline $\begin{array}{l}\text { follicle-stimulating } \\
\text { hormone }(\mathrm{mlU} / \mathrm{ml})\end{array}$ & $6.57 \pm 0.79$ & $6.67 \pm 0.68$ & 0.486 \\
\hline $\begin{array}{l}\text { total testosterone } \\
(\mathrm{ng} / \mathrm{ml})\end{array}$ & $0.94 \pm 0.09$ & $0.99 \pm 0.12$ & $<0.05$ \\
\hline estradiol (pg/ml) & $65.22 \pm 12.86$ & $61.35 \pm 13.66$ & 0.141 \\
\hline LAP & $26.8 \pm 12.8$ & $95.2 \pm 28.9$ & $<0.001$ \\
\hline
\end{tabular}

Table 2 The area under ROC for LAP, WC, BMI and TG to identify MS in PCOS women.

\begin{tabular}{|llll|} 
Markers & $\begin{array}{l}\text { Area under } \\
\text { ROC curve }\end{array}$ & $\begin{array}{l}\text { 95\% Confi- } \\
\text { dence interval }\end{array}$ & P-values \\
\hline LAP & $0.991 \pm 0.006$ & $0.980-1.022$ & $<0.001$ \\
\hline WC & $0.975 \pm 0.015$ & $0.945-1.010$ & $<0.001$ \\
\hline BMI & $0.939 \pm 0.019$ & $0.916-0.971$ & $<0.001$ \\
\hline TC & $0.908 \pm 0.028$ & $0.853-0.962$ & $<0.001$ \\
\hline
\end{tabular}

of Suzhou University were enrolled. PCOS was diagnosed according to the criteria of Rotterdam 2003 [15]. The age of patients ranged from 18 to 34 years (average age: 24.8 years), who had no history of drugs affecting sex hormone, glucose and lipid metabolism. Studies were performed on the $2^{\text {nd }}$ to the $5^{\text {th }}$ day of the menstrual cycle in PCOS women with spontaneous menses and arbitrary dates in PCOS women with amenorrhea. This study was conducted with approval from the Ethics Committee of the Third Affiliated Hospital of Suzhou University. Written informed consent was obtained from all participants.

Patients were defined as having MS, based on International Diabetes Federation (IDF) criteria for MS, if they had central obesity (waist circumference $\geq 80 \mathrm{~cm}$ ) plus 2 or more of the following 4 factors: i) increased concentration of triglycerides (TG): $\geq 1.7 \mathrm{mmol} / \mathrm{l}$, ii) reduced concentration of high-density lipoprotein cholesterol $($ HDL-C): $\leq 1.29 \mathrm{mmol} / \mathrm{l}$, iii) raised blood pressure: systolic pressure $\geq 130 \mathrm{mmHg}$ or diastolic pressure $\geq 85 \mathrm{mmHg}$ or treatment of previously diagnosed hypertension, and iv) increased fasting glucose (FG) level $\geq 5.6 \mathrm{mmol} / \mathrm{l}$ (impaired FG, IFG) or previously diagnosed type 2 diabetes.

\section{Research methods}

All PCOS patients underwent anthropometric measurements, including weight, height, waist circumferences (WC), Systolic blood pressure (SBP), Diastolic blood pressure (DBP) and the body mass index (BMI) was calculated. Fasting blood samples were obtained from PCOS patients in order to measure luteinizing hormone ( $\mathrm{LH}$ ), follicle-stimulating hormone (FSH), estradiol (E2), total testosterone ( $\mathrm{T}$ ), fasting glucose (FBG), fasting insulin (FINS), serum triglycerides (TG), total cholesterol (TC), high density lipoprotein (HDL-C) and low density lipoprotein (LDL-C) levels.

A standard 75-g oral glucose tolerance test was performed for the evaluation of the glucose tolerance status. Insulin sensitivity was assessed by homeostasis model assessment of insulin resistance $($ HOMA-IR), HOMA-IR=FBG $(\mathrm{mmol} / \mathrm{L}) \times$ FINS $(\mathrm{mIU} / \mathrm{L}) / 22.5$. LAP was calculated using the formula [waist $(\mathrm{cm})-58$ ] $\times$ triglyceride concentration $(\mathrm{mmol} / \mathrm{l})$, as previously reported [10].

\section{Statistical analysis}

Statistical analysis was performed by using SPSS15.0 statistical software. Results are presented as mean \pm SD. Prevalence rates are expressed as percentages. Independent samples $t$-test was used for comparison of continuous variables between PCOS women with MS and those without MS. Receiver operating characteristic (ROC) curves analysis was performed for LAP, BMI, TG and WC to identify MS in PCOS women. Areas under the curve (AUC) of the ROC curves and their 95\% confidence intervals (CI) were evaluated as a measure of diagnostic accuracy. The correlation between variables was tested using the 2-tailed Spearman rank correlation test, a $P$-values less than 0.05 was considered statistically significant.

\section{Results}

$\nabla$

Among 105 PCOS women, 45 subjects had MS, and the prevalence of MS was $42.8 \%$ in this study. As shown in $\odot$ Table 1, women with and without MS were similar in age and did not differ significantly in their levels of LDL-C, FSH and E2. PCOS women with MS had significantly increased BMI, WC, SBP, DBP, FBG, postload glucose (PBG), TG and HOMA-IR compared to those without MS. In addition, HDL cholesterol levels were significantly lower in women with MS. Finally, T and LH was significantly higher in PCOS women with MS compared to those without MS ( Table 1).

PCOS women with MS had significantly higher LAP levels $(95.2 \pm 28.9$ vs. $26.8 \pm 12.8)$ compared to those without MS (॰ Table 1).

There was a significant positive correlation between LAP and WC $(r=0.880, p<0.001)$, SBP $(r=0.601, p<0.001)$, DBP $(r=0.564$, $p<0.001)$, FBG $(r=0.479, p<0.001)$, PBG $(r=0.517, p<0.001)$, TG $(r=0.910, p<0.001)$, HOMA-IR $(r=0.597, p<0.001)$ respectively. In addition, there was a significant negative correlation between LAP and HDL-C $(r=-0.691, p<0.001)$.

The results of LAP, WC, BMI and TG ROC curve analysis showed that each marker is a significant discriminator for MS in PCOS women. The largest AUC was obtained with LAP, indicating that LAP was superior for estimating the MS of PCOS patients in this study ( $\bullet$ Table 2 ). From the ROC curve analysis, the optimal cutoff points of LAP to predict MS in PCOS were 54.2 (93.3\% sensitivity, 96.7\% specificity, ○ Fig. 1). 


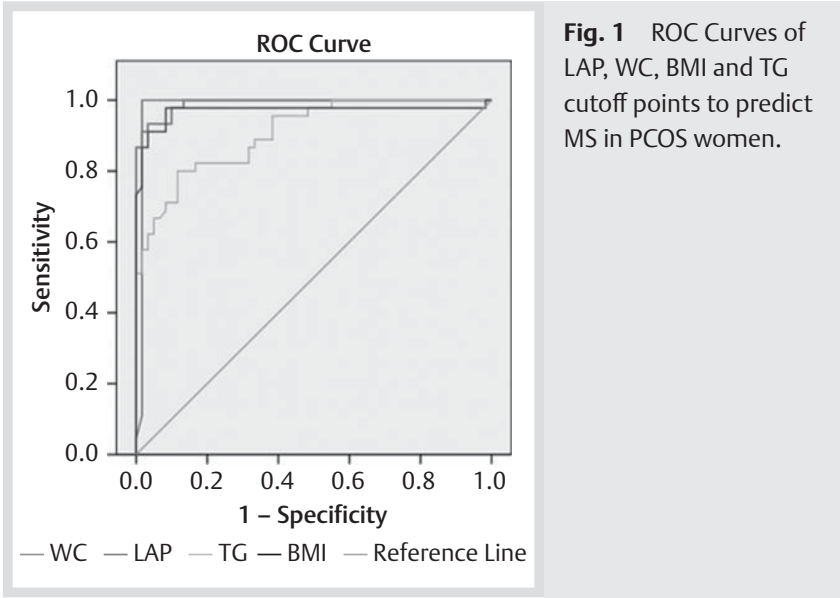

\section{Discussion}

PCOS is a condition associated with increased risk for cardiovascular disturbances $[16,17]$. PCOS women are at increased risk of having MS, and the prevalence of MS in PCOS patients varies among different ethnicities depends on the definition used [1820]. In this study, the prevalence of MS, as diagnosed according to IDF criteria, was $42.8 \%$ in the studied Chinese PCOS patients. MS itself is a risk for type 2 diabetes and CVD, so early and accurate identification of high-risk individuals for MS is of great importance [21].

Previous studies have evaluated enlarged WC and elevated TG in different populations as a surrogate marker of cardiovascular risk. LAP, an ordinal scale combining WC and TG, could be associated to a dysfunctional and highly lipolytic adipose tissue that is a central abnormality behind MS, CVD and type 2 diabetes [22]. The population-based United States' National Health and Nutrition Examination Survey (NHANES III) concluded that LAP was superior to BMI for recognizing CVD risk and diabetes $[10,11]$. In the present study, PCOS women with MS had higher LAP levels in comparison to those without MS. This indicates that high LAP levels are associated with MS in PCOS women. IR plays a central role in both the reproductive and metabolic disturbances observed in women with PCOS [23]. In our study, PCOS women with MS had significantly increased HOMA-IR compared to those without MS. Meantime, we showed that LAP was significantly correlated with HOMA-IR, indicating that LAP may be useful to precociously screen a subset of young women who are susceptible to the development of IR-related comorbidities, including MS, diabetes and CVD. In addition, we demonstrated LAP was highly correlated with WC, SBP, DBP, FBG, PBG, TG and HDL-C, which were components of MS. Therefore, LAP is related to MS and can serves as a reliable marker of MS in PCOS women [24].

Both WC and BMI are shown to be good predictors of the presence of MS [25]. TG, also, is a reliable predictor for these cardiometabolic syndromes. In our study, ROC analysis showed that LAP, WC, BMI and TG were significant discriminators for MS in PCOS women. However, the largest AUC was obtained with LAP, indicating that LAP was superior to WC, BMI and TG for predicting MS of PCOS patients. In addition, ROC curve showed that $\mathrm{LAP} \geq 54.2$ had adequate sensitivity and specificity for detecting a state of MS in PCOS women. The high sensitivity and specificity of LAP detected in ROC curve analysis indicate that LAP might be a useful marker to predict MS in PCOS women. The early identi- fication of PCOS women with MS and introduction of therapeutic interventions may help delay the progression to CVD and T2DM.

Limitations of the present study are the small sample size and the ethnic origin, Therefore, further research should be undertaken in larger sample sizes and different ethnic groups.

In conclusion, our results show that LAP, an easily obtainable measure, has a strong and reliable diagnostic accuracy for MS in PCOS women. Early and accurate identification of high-risk individuals for MS will allow introduction of therapeutic interventions to prevent CVD and type 2 diabetes.

\section{Acknowledgements}

\section{$\nabla$}

We thanked the patients for participating in this study. We are grateful to Dr. Qing Mao for critical reading of this manuscript.

Declaration of interests: The authors declare no conflicts of interest.

\section{References}

1 Azziz R, Woods KS, Reyna $R$ et al. The prevalence and features of the polycystic ovary syndrome in an unselected population. J Clin Endocrinol Metab 2004; 89: 2745-2749

2 Ehrmann DA. Polycystic ovary syndrome. N Engl J Med 2005; 352: 1223-1236

3 Homburg $R$. Polycystic ovary syndrome. Best Pract Res Clin Obstet Gynaecol 2008; 22: 261-274

4 Dunaif $A$, Xia J, Book $C B$ et al. Excessive insulin receptor serine phosphorylation in cultured fibroblasts and in skeletal muscle. A potential mechanism for insulin resistance in the polycystic ovary syndrome. J Clin Invest 1995; 96: 801-810

5 Gambineri A, Pelusi C, Vicennati $V$ et al. Obesity and the polycystic ovary syndrome. Int J Obes Relat Metab Disord 2002; 26: 883-896

6 Lo JC, Feigenbaum SL, Yang $J$ et al. Epidemiology and adverse cardiovascular risk profile of diagnosed polycystic ovary syndrome. J Clin Endocrinol Metab 2006; 91: 1357-1363

7 Coviello $A D$, Legro $R S$, Dunaif $A$. Adolescent girls with polycystic ovary syndrome have an increased risk of the metabolic syndrome associated with increasing androgen levels independent of obesity and insulin resistance. J Clin Endocrinol Metab 2006; 91: 492-497

8 Ford ES. The metabolic syndrome and mortality from cardiovascular disease and all-causes: findings from the National Health and Nutrition Examination Survey II Mortality Study. Atherosclerosis 2004; 173: 309-314

9 Rossi B, Sukalich S, Droz J et al. Prevalence of metabolic syndrome and related characteristics in obese adolescents with and without polycystic ovary syndrome. J Clin Endocrinol Metab 2008; 93: 4780-4786

10 Kahn HS. The "lipid accumulation product" performs better than the body mass index for recognizing cardiovascular risk: a populationbased comparison. BMC Cardiovasc Disord 2005; 5: 26

11 Kahn HS. The lipid accumulation product is better than BMI for identifying diabetes: a population-based comparison. Diabetes Care 2006; 29: 151-153

12 Wehr E, Gruber HJ, Giuliani A et al. The lipid accumulation product is associated with impaired glucose tolerance in PCOS women. J Clin Endocrinol Metab 2011; 96: E986-E990

13 Taverna MJ, Martinez-Larrad MT, Frechtel GD et al. Lipid accumulation product: a powerful marker of metabolic syndrome in healthy population. Eur J Endocrinol 2011; 164: 559-567

14 Tellechea ML, Aranguren F, Martinez-Larrad MT et al. Ability of lipid accumulation product to identify metabolic syndrome in healthy men from Buenos Aires. Diabetes Care 2009; 32: e85

15 Revised 2003 consensus on diagnostic criteria and long-term health risks related to polycystic ovary syndrome. Fertil Steril 2004; 81: 19-25

16 Cussons AJ, Watts GF, Burke V et al. Cardiometabolic risk in polycystic ovary syndrome: a comparison of different approaches to defining the metabolic syndrome. Hum Reprod 2008; 23: 2352-2358 
17 Cheung $L P$, Ma RC, Lam PM et al. Cardiovascular risks and metabolic syndrome in Hong Kong Chinese women with polycystic ovary syndrome. Hum Reprod 2008; 23: 1431-1438

18 Verit FF. The prevalence of metabolic syndrome in clomiphene citrate resistant polycystic ovary syndrome. Gynecol Endocrinol 2012; 28: 365-369

19 Bhattacharya SM. Prevalence of metabolic syndrome in women with polycystic ovary syndrome, using two proposed definitions. Gynecol Endocrinol 2010; 26: 516-520

$20 \mathrm{Ni}$ RM, Mo Y, Chen X et al. Low prevalence of the metabolic syndrome but high occurrence of various metabolic disorders in Chinese women with polycystic ovary syndrome. Eur J Endocrinol 2009; 161: 411-418

21 OhJY, Sung YA, Lee HJ et al. Optimal waist circumference for prediction of metabolic syndrome in young Korean women with polycystic ovary syndrome. Obesity (Silver Spring) 2010; 18: 593-597
22 Despres JP, Lemieux I, Bergeron J et al. Abdominal obesity and the metabolic syndrome: contribution to global cardiometabolic risk. Arterioscler Thromb Vasc Biol 2008; 28: 1039-1049

23 Wild S, Pierpoint T, McKeigue P et al. Cardiovascular disease in women with polycystic ovary syndrome at long-term follow-up: a retrospective cohort study. Clin Endocrinol (Oxf) 2000; 52: 595-600

24 Wiltgen D, Benedetto IG, Mastella LS et al. Lipid accumulation product index: a reliable marker of cardiovascular risk in polycystic ovary syndrome. Hum Reprod 2009; 24: 1726-1731

25 Wang $F$, Wu S, Song $Y$ et al. Waist circumference, body mass index and waist to hip ratio for prediction of the metabolic syndrome in Chinese. Nutr Metab Cardiovasc Dis 2009; 19: 542-547 